\title{
Multiple system atrophy
}

\author{
H U Rehman
}

Multiple system atrophy (MSA) is a degenerative disease of the central nervous system. Dejerine and Thomas in 1900 were the first to use the term olivopontocerebellar atrophy (OPCA) in two sporadic cases, ${ }^{1}$ although Menzel described the first case in 1891. Shy and Drager in 1960 described four cases of a "neurological syndrome with orthostatic hypotension". 2 They defined the full syndrome comprising of orthostatic hypotension, urinary and rectal incontinence, loss of sweating, iris atrophy, external ocular palsies, rigidity, tremor, loss of associated movements, impotence, atonic bladder, loss of rectal sphincter tone, fasciculations, wasting of distal muscles, evidence of a neuropathic lesion in the electromyogram suggesting involvement of the anterior horn cells, and the finding of a neuropathic lesion in the muscle biopsy. Adams et al first described a syndrome designated striatonigral degeneration in the early 1960s. ${ }^{3}$ These patients had mild autonomic failure and ataxia and were shown to have lesions in the olivopontocerebellar system. Graham and Oppenheimer first proposed the term multiple system atrophy in 1969 and suggested that OPCA, idiopathic orthostatic hypotension, the Shy-Drager syndrome, and striatonigral degeneration "are merely the expression of neuronal atrophy in a variety of overlapping combinations". 4

MSA is a sporadic disease. MSA was considered a rare disease but many patients with MSA are misdiagnosed as suffering from idiopathic Parkinson's disease. It is clear from a large postmortem series of parkinsonian brains that cases of MSA are more common than previously thought. Between $4 \%$ and $22 \%$ of the brains in parkinsonian brain banks have MSA. ${ }^{5}$ According to one estimate the prevalence of MSA is 16.4 per 100000 population. Mean age of disease onset is 53 years (range 36-74) and the median disease duration to death is five years (range 1-11). Both sexes are affected equally. ${ }^{6}$

\section{Clinical features}

MSA can cause any combination of parkinsonian, autonomic, pyramidal, and cerebellar signs. When parkinsonian features predominate, the term striatonigral degeneration is often used. When cerebellar features predominate, sporadic OPCA is often used. When autonomic failure predominates, the term ShyDrager syndrome is often used. These manifestations may occur in various combinations and evolve with time.

Most patients with MSA are initially considered to have Parkinson's disease (see box for features that raise doubts about the diagnosis). Parkinsonian features are documented in 91\%
"Red flags"-clinical features raising doubts about the diagnosis of idiopathic Parkinson's disease

- Poor or absent levodopa response.

- Lack of typical levodopa induced dyskinesias.

- Atypical levodopa induced dyskinesiasfor example, sustained dystonic spasm of facial muscles, torticollis, antecollis.

- Pyramidal signs.

- Cerebellar signs.

- Autonomic failure.

- Early falls and instability.

- Rapid clinical deterioration despite dopaminergic treatment.

- Myoclonic jerks.

- Severe dysarthria.

- Pains not relieved by levodopa.

of the patients. Akinesia and rigidity predominate and is usually asymmetric. Tremor may be at rest, jerky irregular, or a classic pill rolling.

Autonomic symptoms are present in $97 \%$ of patients. They may precede the motor disturbance by months or even years. Impotence is almost universal in male patients. Postural hypotension is usually mild to moderate. It may cause recurrent syncopal attacks. Urinary urge incontinence and urinary retention may also occur.

Cerebellar signs occur in about half the patients. MSA of OPCA type most commonly presents with gait ataxia. Pyramidal signs, a mixed dysarthria with combination of hypokinetic, ataxic and spastic components, ${ }^{7}$ nystagmus, saccadic pursuit, hypometric saccades, limitation of gaze, myoclonus, and impaired distal vibration sense and joint position sense are sometimes seen. Dysphagia may become significant in the later part of the illness. Respiratory stridor may also occur. This is caused by atrophy of the posterior cricoarytenoid muscles resulting in failure of abduction of the vocal cords and narrowing of the airway. ${ }^{8}$

In the fully developed disease they are permanently bedbound, almost mute and severely dysphagic with an indwelling catheter, yet with retained intellect and awareness.

The cause of death in MSA is commonly related to development of bulbar dysfunction and dysphagia, predisposing to aspiration pneumonia.

\section{Differential diagnosis}

MSA is most commonly misdiagnosed as idiopathic Parkinson's disease. Asymmetry, rest tremor, and a good levodopa response are suggestive of idiopathic Parkinson's disease, but do not exclude MSA. A poor or absent levodopa response is the rule in MSA patients, although transient but excellent benefit may occur. ${ }^{9}$
Submitted 12 July 2000 Accepted 28 November 2000 
Many patients, however, may appear to be deriving no benefit from their therapy, yet deteriorate hours or days after stopping it. ${ }^{10}$ Patients with MSA on chronic levodopa treatment lack typical, or display atypical, levodopa induced dyskinesias. These can take form of sustained dystonic spasms of facial muscles, ${ }^{11}$ torticollis, or a relatively fixed and disproportionate antecollis. ${ }^{12}$

Although a few patients may display inappropriate laughter or crying, intellect is strikingly preserved. ${ }^{6}$ Therefore dementia is not a feature of MSA. ${ }^{13}$ Presence of pyramidal or cerebellar signs or autonomic failure should raise a suspicion of MSA. Instability and falls early in the course of the disease should also suggest a cause other than idiopathic Parkinson's disease. Rapid clinical deterioration despite dopaminergic treatment is another clue. Myoclonic jerks, often stretch sensitive, usually affecting the fingers is rare in idiopathic Parkinson's disease. ${ }^{14}$ Speech is more severely affected in MSA than in idiopathic Parkinson's disease. Deep aching limb pains occur in both idiopathic Parkinson's disease and MSA, but unlike the pain of idiopathic Parkinson's disease, it is not relieved by levodopa in MSA. ${ }^{15}$

Another important differential diagnosis is pure autonomic failure. Other than clinical manifestations, plasma noradrenaline (norepinephrine) concentrations in the supine resting condition may help to differentiate the two conditions. Plasma noradrenaline, an index of peripheral sympathetic activity, is often normal in patients with MSA, reflecting intact peripheral sympathetic neurones, but abnormally low in patients with pure autonomic failure, indicating abnormal peripheral sympathetic neurones. ${ }^{16}$ However, the distinction on the basis of plasma noradrenaline has been unreliable. ${ }^{17}$ Kaufmann et al therefore proposed that plasma arginine vasopressin (AVP) measurements in upright tilt are a more sensitive way to differentiate the two conditions. ${ }^{18}$ In patients with MSA upright tilt produced a marked fall in blood pressure, but plasma AVP increased only slightly and inappropriately for the degree of hypotension. In patients with pure autonomic failure, however, a similar degree of hypotension to that in MSA patients, elicited a marked rise in circulating AVP.

\section{Pathology}

Macroscopically, changes in MSA are generally related to the clinical pattern of the disease. ${ }^{19}$ There may be variable cortical atrophy, although in most cases this is not marked. Atrophy of cerebellum, middle cerebellar peduncles, and the pons are most marked in patients with the OPCA variant of the disease. There are varying degrees of cell loss and gliosis, without Lewy bodies in the substantia nigra, locus coeruleus, striatum, inferior olives, pontine nuclei and cerebellar Purkinje's cells, and in the intermediolateral cell columns and Onuf's nucleus in the ventral horn of the spinal cord at second and third sacral levels. ${ }^{19}$ Loss of neurones in the intermediolateral column of the spinal cord relates to the autonomic disturbances in $\mathrm{MSA}^{20}$ Dorsal motor nucleus of vagus, vestibular nuclei, pyramidal tracts, and anterior horns may also be affected. Other medullary nuclei affected include catecholaminergic cells in the ventrolateral reticular formation $^{21}$ and cells in the arcuate nucleus. The absence of Lewy bodies distinguishes it from idiopathic Parkinson's disease. A useful macroscopically diagnostic clue is a greygreenish discoloration of the atrophic putamen. The substantia nigra and locus coeruleus are pale as a result of loss of pigment.

There are five histological features, which characterise MSA: glial cytoplasmic inclusions, neuronal cytoplasmic inclusions, neuronal nuclear inclusions, glial nuclear inclusions, and neurophil threads. Glial cytoplasmic inclusions (GCIs) are the most important. GCIs have been recently described in MSA with various combinations of striatonigral degeneration, OPCA, and Shy-Drager syndrome. ${ }^{22}$ These inclusions are not specific, although they are characteristic of MSA. This also confirms the notion that the three clinical syndromes of striatonigral degeneration, OPCA, and ShyDrager syndrome are various manifestations of the same disease. GCIs have become a diagnostic hallmark of MSA. GCIs are found in the primary motor and higher motor areas of the cerebral cortex, pyramidal, extrapyramidal, and corticocerebellar systems and in the supraspinal autonomic systems and their targets. These inclusions occur in oligodendrocytes and may precede neuronal alteration, implying that oligodendroglial degeneration and not the degeneration of axons or neuronal cell bodies is a prerequisite for the development of GCIs and that GCIs are unlikely to have developed as a reaction to axonal injury. ${ }^{23}$ Immunohistochemical studies have shown that GCIs contain $\alpha$-synuclein, a synaptic protein also found in Lewy bodies in Parkinson's disease.$^{24}$ It has been suggested that a reduction in the solubility of $\alpha$-synuclein may induce this protein to form filaments that aggregate into cytoplasmic inclusions thus contributing to the dysfunction or death of glial cells.

\section{Neurochemistry}

Various changes in the dopaminergic, noradrenergic, serotoninergic, and cholinergic systems have been described. Changes in the dopaminergic system include loss of dopamine; its synthetic enzyme, tyrosine hydoxylase; and a cofactor for tyrosine hydroxylase, tetrahydrobiopterin. Noradrenaline concentrations have been reported to be low in the locus coeruleus and hypothalamus. Severe $(>75 \%)$ loss of catecholaminergic neurones in the ventrolateral medulla, particularly in the rostral ventrolateral medulla, which contains the $\mathrm{Cl}$ adrenergic neurones that provide tonic vasomotor input to the sympathetic preganglionic neurones, has been reported. ${ }^{21}$

Concentrations of homovanillic acid, the major metabolite for dopamine, 3-methoxy-4hydroxyphenylglycol, the major metabolite for noradrenaline and 5-hydroxyindoleacetic acid, the major metabolite of serotonin is low in the cerebrospinal fluid. ${ }^{25}$ 


\section{Investigations}

Cardiovascular autonomic function tests can demonstrate various aspects of autonomic failure. The plasma noradrenaline concentrations are normal or slightly raised in MSA patients differentiating it from pure autonomic failure in which plasma noradrenaline is usually 1 ow. ${ }^{17}$ However, the clinical usefulness of plasma noradrenaline concentrations as a diagnostic tool in individual cases has not been demonstrated.

Computed tomography is usually normal in patients presenting a predominantly a parkinsonian syndrome. In subjects with a predominantly cerebellar presentation, cerebellar and/or brainstem atrophy may be seen but this is non-specific. Striatal computed tomography images are normal in all forms of MSA. Computed tomography is only of limited usefulness in the diagnosis of MSA unlike magnetic resonance imaging which may show altered signal in striatum, particularly in putamen, thus differentiating it from idiopathic Parkinson's disease. Magnetic resonance imaging may show putaminal atrophy on T2 weighted images and a hyperintense rim at the lateral putaminal edge, often accompanied by putaminal hypointensity. ${ }^{26}$

Electrophysiological monitoring of individual motor units from the striated component of urethral sphincter has shown consistent abnormalities in MSA patients, reflecting loss of anterior horn cells in Onuf's nucleus in the sacral cord and is highly specific (0.92) but less sensitive (0.62) ${ }^{27}$ Although occasional patients with incontinence have normal studies, subclinical abnormalities can also be detected.

\section{Treatment}

Treatment is mainly supportive. Levodopa preparations may transiently improve the symptoms but usually are ineffective. ${ }^{28}$ Head-up tilt of the bed at night, elastic support stockings, fludrocortisone, and desmopressin (DDAVP) may help the postural hypotension. ${ }^{29}$ Octreotide inhibits release of vasodilator peptides and may be useful in postural hypotension, although it must be given subcutaneously. ${ }^{25} \mathrm{~A}$ number of vasoconstrictors can also be used. These include midodrine, a peripherally acting $\alpha_{1}$-agonist, as well as ephedrine and phenylpropanolamine. ${ }^{25}$

Anticholinergics can help to reduce urinary frequency but may precipitate urinary retention. Retention with overflow may require bethanecol chloride, a cholinergic muscarinic agonist, ${ }^{30}$ intermittent self catheterisation, or an indwelling catheter. Constipation may be helped by a high fibre diet and bulk laxatives or may require suppositories or enemas.

Impotence may be treated with intracavernosal injections of papaverine, prostaglandin $\mathrm{E}$, or implantation of a penile prosthesis. ${ }^{31}$ Sildenafil has not been systematically evaluated in patients with MSA. Tracheostomy or laser cricoarytenoidectomy may be considered for stridor, but wishes of the patient and ethical considerations will influence the decision. ${ }^{31}$

\section{Questions (answers on p 382)}

1. The following statements are true about multiple system atrophy:

(A) MSA is a sporadic disease.

(B) Between $4 \%$ and $22 \%$ of the brains in parkinsonian brain banks have MSA.

(C) The prevalence of MSA is 8.6 per 100000 population.

(D) Mean age of disease onset is 68 years and the median disease duration to death is two years.

(E) Both sexes are affected equally.

2. The following statements are true:

(A) Autonomic symptoms are present in $60 \%$ of patients.

(B) Autonomic symptoms may precede the motor disturbance by months or even years.

(C) Cerebellar signs occur in $80 \%$ of the patients.

(D) Dysphagia is an early feature of the illness.

(E) Akinesia and rigidity is usually symmetric.

3. Which of the following statements are true? (A) A poor or absent levodopa response is the rule in MSA patients.

(B) Intellect is impaired early in the course of MSA.

(C) Deep aching limb pains are not

relieved by levodopa in MSA.

(D) Instability and falls early in the course of the disease are characteristic of idiopathic Parkinson's disease.

(E) Patients with MSA and idiopathic Parkinson's disease on chronic levodopa treatment are equally likely to have sustained dystonic spasms of facial muscles, torticollis, or a relatively fixed and disproportionate antecollis.

4. The following are true about the histological and biochemical features of MSA:

(A) The absence of Lewy bodies distinguishes it from idiopathic Parkinson's disease.

(B) A reddish-blue discoloration of the atrophic putamen.

(C) GCIs are specific for MSA.

(D) Changes in the dopaminergic system include loss of dopamine.

(E) Noradrenaline concentrations are high in the locus coeruleus and hypothalamus.

5. Which of the following statements about investigations of MSA are true?

(A) The plasma noradrenaline concentrations are normal or slightly raised in MSA. (B) Magnetic resonance imaging is of no use in the diagnosis of MSA.

(C) Electrophysiological monitoring of individual motor units from the striated component of urethral sphincter have a low specificity in the diagnosis of MSA.

(D) Computed tomography is only of limited usefulness in the diagnosis of MSA.

(E) Striatal CT images are normal in all forms of MSA. 
Physiotherapy, occupational therapy, and speech therapy can prove very useful and can provide substantial benefit in activities of daily living.

\section{Summary}

MSA is a degenerative disease of the central nervous system. It is now recognised that MSA encompasses a variety of clinical syndromes and OPCA, idiopathic orthostatic hypotension, the Shy-Drager syndrome, and striatonigral degeneration are merely the expression of neuronal atrophy in a variety of overlapping combinations. MSA can cause any combination of parkinsonian, autonomic, pyramidal, and cerebellar signs. Treatment is mainly supportive, as no curative therapy is known. Multiple system atrophy is most commonly confused with idiopathic Parkinson's disease. Knowledge of the distinguishing features of the two conditions is important for an accurate diagnosis and from a prognostic point of view.

1 Dejerine J, Thomas AA. L'atrophie olivo-pontocerebelleuse. Nouv Iconog de le Salpetriere 1900;13:330-70.

2 Shy GM, Drager GA. A neurologic syndrome associated with orthostatic hypotension. Arch Neurol 1960;2:511-27.

3 Adams RD, Van Bogaert L, Van der Eecken H. Striatonigral degeneration. F Neuropathol Exp Neurol 1964;23:584608 .

4 Graham JG, Oppenheimer DR. Orthostatic hypotension and nicotine sensitivity in a case of multiple system atrophy. f Neurol Neurosurg Psychiatry 1969;32:28-34.

5 Ouinn N, Wenning G. Multiple system atrophy. Br f Hosp Quinn N, Wenning

6 Quinn N. Multiple system atrophy-the nature of the beast. f Neurol Neurosurg Psychiatry 1989; $\mathrm{S}$ suppl:78-89.

7 Karen J, Kinin MS, Gilman S, et al. Characteristics of the dysarthria of multiple system atrophy. Arch Neurol 1996;53: $545-8$

8 Guindi GM, Michaels L, Bannister R, et al. Pathology of the intrinsic muscles of the larynx. Clin Otolaryngol 1981;6:101-

9 Wenning GK, Ben Shlomo Y, Magalhaes M, et al. Clinica features and natural history of multiple system atrophy. An analysis of 100 cases. Brain 1994;117:835-45.

10 Hughes AJ, Colosimo C, Kleedorfer B, et al. The dopaminergic response in multiple system atrophy. $f$ Neuro Neurosurg Psychiatry 1992;55:1009-13.

11 Quinn NP. Unilateral facial dystonia in multiple system atrophy. Mov Disord 1992;7(suppl 1):79.

12 Quinn N. Disproportionate antecollis in multiple system atrophy. Lancet 1989;i:844.

13 Robbins TW, James M, Lange KW, et al. Cognitive performance in multiple system atrophy. Brain 1992;115: 271-91

14 Obeso JA, Rodriguez ME, Artieda J, et al. Focal reflex myoclonus: a useful sign in the differential diagnosis of parkinsonism. Ann Neurol 1989;26:164-5.

15 Caplan LR. Clinical features of sporadic (DejerineThomas) olivopontocerebellar atrophy. Adv Neurol 1984;41: 217-24

16 Ziegler MG, Lake CR, Kopin IJ. The sympathetic nervous system defect in primary orthostatic hypotension. $\mathrm{N} \mathrm{Engl}$ Med 1977;296:293-7.

17 Polinsky RJ. Neurotransmitter and neuropeptide function in autonoruic failure. In: Bannister R, ed. Autonomic failure. A textbook of clinical disorders of the autonomic nervous system. 2nd Ed. Oxford: Oxford University Press, 1988: 321-47.

18 Kaufmann H, Oribe E, Miller M, et al. Hypotensioninduced vasopressin release distinguishes between pure autonomic failure and multiple system atrophy with autonomic failure. Neurology 1992;42:590-3.

19 Oppenheimer DR. Neuropathology of progressive autonomic failure. In: Bannister R, ed. Autonomic failure. A textbook of clinical disorders of the autonomic nervous system. 2nd Ed. Oxford: Oxford University Press, 1988: 267-83.

20 Kennedy PG, Duchen LW. A quantitative study of intermediolateral column cells in motor neuron disease and the Shy-Drager syndrome. F Neurol Neurosurg Psychiatry 1985; 48:1103-6.

21 Benarroch EE, Smithson IL, Low PA, et al. Depletion of catecholaminergic neurons of the rostral ventrolateral medulla in multiple system atrophy with autonomic failure. Ann Neurol 1998;43:156-63.

22 Papp MI, Kahn JE, Lantos PL. Glial cytoplasmic inclusions in the CNS of patients with multiple system atrophy (striatonigral degeneration, olivopontocerebellar atrophy

3 Lantos PL, Papp MI. Cellular pathology of multiple system atrophy: a review. F Neurol Neurosurg Psychiatry 1994;57: $129-33$.

$24 \mathrm{Tu} \mathrm{PH}$, Galvin JE, Baba M, et al. Glial cytoplasmic inclusions in white matter oligodendrocytes of multiple system atrophy brains contain insoluble alpha-synuclein. Ann Neurol 1998;44:415-22.

25 Mathias CT, Williams AC. The Shy-Drager syndrome (and multiple system atrophy). In: Caine DB, ed. Neurodegenerative diseases. Philadelphia: WB Saunders, 1994: 743-67.

26 Olanow CW. Magnetic resonance imaging in parkinsonism. Neurol Clin 1992;10:405-20.

27 Eardley I, Quinn NP, Fowler CT, et al. The value of urethral sphincter electromyography in the differential diagnosis of parkinsonism. Br F Urol 1989;64:360-2.

28 Lees AJ. The treatment of multiple system atrophy: striatonigral degenerationand olivopontocerebeller degeneration. In: Bannister R, ed. Autonomic failure. A textbook of clinical disorders of the autonomic nervous system. 2nd Ed. Oxford: Oxford University Press, 1988: 596-604

29 McLeod JG, Tuck RR. Disorders of the autonomic nervous system: part 2. Investigation and treatment. Ann Neurol 1987;21:519-29.

30 Fazzini E. Multiple system atrophy associated with progressive autonomic failure. The Shy-Drager syndrome: a new classification scheme. In. Stem MB, Koller WC, eds Parkinsonian syndromes. New York: Marcel Dekker, 1993: 69-78.

31 Quinn N. Multiple system atrophy. In: Marsden CD, Faun $\mathrm{S}$, eds. Movement disorders 3. Oxford: ButterworthHeinemann, 1994: 262-81.

\section{Answers}

1. (A) true; (B) true; (C) false: the true prevalence of MSA is 16.4 per 100000 population; (D) false: the mean age of onset is 53 years (range 36-74) and the median disease duration to death is five years (range $1-11$ ); (E) true.

2. (A) false: autonomic symptoms are present in $97 \%$ of cases; (B) true; (C) false: cerebellar signs occur in about half the patients; (D) false: dysphagia may become significant in the later part of the illness; (E) false: akinesia and rigidity is usually asymmetric.

3. (A) true; (B) false: intellect is strikingly preserved, therefore dementia is not a feature of MSA; (C) true; (D) false: instability and falls early in the course of the disease should also suggest a cause other than idiopathic Parkinson's disease; (E) false: these features suggest a diagnosis of MSA.

4. (A) true; (B) false: a grey-greenish discoloration of the atrophic putamen; (C) false: GCIs are not specific, although characteristic for MSA; (D) true; (E) false: noradrenaline concentrations have been reported to be low in the locus coeruleus and hypothalamus.

5. (A) false: the plasma noradrenaline concentrations are normal or slightly raised in MSA patients differentiating it from pure autonomic failure in which the plasma noradrenaline concentrations are usually low; (B) false: magnetic resonance imaging may show altered signal in striatum, particularly in putamen, thus differentiating it from idiopathic Parkinson's disease; (C) false: electrophysiological monitoring of individual motor units from the striated component of urethral sphincter has shown consistent abnormalities in MSA patients and is highly specific; (D) true; (E) true. 